\title{
Potentially Harmful Maillard Reaction Products in Food and Herb Medicines
}

\author{
Ya-Li Li ${ }^{1},{ }^{1}$ Yan-Zhu Zhu, ${ }^{1}$ Pei-He Zheng, ${ }^{1}$ Zheng-Yi Qu, ${ }^{1}$ Hao Zhang, ${ }^{1}$ Wei Hou $\left(\mathbb{D},{ }^{1}\right.$ \\ Xiang-Min Piao, ${ }^{2}$ and Ying-Ping Wang ${ }^{2}$ \\ ${ }^{1}$ Institute of Wild Economic Animals and Plants of Chinese Academy of Agriculture Sciences, Changchun, Jilin 132122, China \\ ${ }^{2}$ School of Chinese Medicinal Materials, Jilin Agriculture University, Changchun, Jilin 132118, China \\ Correspondence should be addressed to Wei Hou; jilinhouwei@163.com
}

Received 29 February 2020; Revised 10 November 2020; Accepted 15 December 2020; Published 5 January 2021

Academic Editor: M rcio Carocho

Copyright (c) $2021 \mathrm{Ya}-\mathrm{Li} \mathrm{Li}$ et al. This is an open access article distributed under the Creative Commons Attribution License, which permits unrestricted use, distribution, and reproduction in any medium, provided the original work is properly cited.

\begin{abstract}
The Maillard reaction is of great significance in food, herb medicines, and life processes. It is usually occurring during the process of food and herb medicines processing and storage. The formed Maillard reaction productions (MRPs) in food and herb medicines not only generate a large number of efficacy components but also generate a small amount of harmful substance that cannot be ignored. Some of the MRPs, especially the advanced glycation end products (AGEs) are concerning humans, based on the possibility to induce cancer and mutations in laboratory animals. Numerous studies have been reported on the formation, analysis, and control of the potentially harmful MRPs (PHMRPs). Therefore, the investigation into the formation, analysis, and control of PHMRPs in food and herb medicines is very important for improving the quality and safety of food and herb medicines. This article provides a brief review of the formation, analysis (major content), and control of PHMRPs in food and herb medicines, which will provide a base and reference for safe processing and storage of food and herb medicines. Practical Applications. The formed Maillard reaction productions in food and herb medicines not only generate a large number of functional components but also generate a small amount of harmful substance that cannot be ignored. This contribution provides a brief review on the formation (including the correlative studies between MRs and the PHMRPs, mechanisms, and the main pathways); analysis (major content, pretreatment for analysis, qualitative and quantitative analysis, and structural identification analysis); and control (strategies and mechanisms) of PHMRPs in food and herb medicines, which will provide a solid theoretical foundation and a valuable reference for safe processing and storage for food and herb medicines.
\end{abstract}

\section{Introduction}

The Maillard reaction (MR) is also called the amino-carbonyl reaction, nonenzymatic browning, or protein glycation reaction. It is originally described by Louis Maillard $[1,2]$, concerning a serious of reactions between carbonyl and amino compounds. The MR is occurring in dried, heated, or stored herb medicines, food, and in vivo mammalian organisms. MR usually occurs during the process and storage of food and herb medicines because of the abundant carbonyl and amino compounds contained in them. MR in food and herb medicines processing and storage process not only produces a large number of active ingredients but also generates a small number of potential harm compounds which cannot be ignored. Many contributions reported the formation, analysis, and control of potentially harmful Maillard reaction productions (PHMRPs) in food and herb medicines [3, 4]. Harris et al. [5] reported that some MRPs such as $\varepsilon$-N-2-furoylmethyl-L-lysine (furosine, FML) could degrade to form different advanced glycation end products (AGEs). However, recent evidence shows that MRPs, especially AGEs, have a propensity to generate reactive oxygen species (ROS), and partial MRPs had been proven to be related to some kind of diseases such as Parkinson's disease [6], chronic diabetes [7], Alzheimer's disease [8], and also aging (normal aging) [7, 9]. AGEs in bodies of humans are mainly derived from two pathways, in vitro intake and in vivo transformation, and the intake from food and herb 
medicines is the main source of the formed AGEs [10]. During the process, two types of MRPs can generate, and they can be distinguished by the cross-linking structures and fluorescence properties, depending on the temperature, $\mathrm{pH}$, and characters of the reactants (e.g., type of amino acid, sugar, or protein); one is cross-linking and fluorescent MRPs, such as crossline, FML, 2-(2-furoyl)-4(5)-(2-furanyl)-1H-imidazole (FFI), methyl-glyoxal-lysine dimer (MOLD), glyoxal-lysine dimer (GOLD), fluorolink, pentosidine, and vesperlysines A, B, and C (Figure 1(a)) and another is non-cross-linking and nonfluorescent AGEs, such as argpyrimidine, Ne-(carboxymethyl)lysine (CML), NE(carboxyethyl)lysine (CEL), 3-DG-imidazolones, MG-imidazolones, acrylamide (AA), and pyrraline (Figure 1(b)). The maxima excite wavelengths of $340-370 \mathrm{~nm}$ and emit wavelengths of $420-470 \mathrm{~nm}$ for the compounds concerning the fluorescent MRs; their intensities are related to the level of AGE [11]. The present article reviews the formation, analysis, and control of the MRPs, consisting of formation (pathways and mechanisms), analysis (major content, pretreatment for analysis, qualitative and quantitative analysis, and structural identification analysis) and with an emphasis on control (strategies and mechanisms) of the PHMRPs. The aim of this paper is to search for strategies for preventing or inhibiting the undesired MRPs formation during the storage and processing of food and herb medicines, which will provide a base and reference for the safety of utilization.

\section{PHMRPs Formation and Mitigation}

The MRs are usually concerning three stages: the initial, intermediate, and final stage [12]. So, the obtained MRPs conclude initial MRPs, advanced MRPs, and AGEs (Figure 2). The initial stage starts from a reaction between carbonyl group compounds (such as sugar) and free amino group compounds (e.g., sugars, peptides, or proteins), leading to form an unstable Schiff base, which then generates a stable Amadori product. It is reported that the Schiff base is highly prone to form reactive carbonyl compounds (e.g., oxoaldehydes, glyoxal, and methylglyoxal) [13]. On the other side, reduced sugars such as glucose can autooxidize to form hydrogen peroxide and keto aldehydes in the specific conditions [14] and subsequently to form AGEs. Consequently, the Amadori products react with the amino acids to form CML or regenerate amines and subsequently to form dicarbonyls such as 1-deoxyglucosone (1-DG), 3-DG, and glycolaldehyde. Finally, the final advanced stage of the reaction occurs. In this stage, the highly reactive DG reacts with lysine or lysine residues in proteins to form pralines. Meanwhile, cross-linking and fragmentation formed in the protein molecule, leading to protein denaturation and damage. During the process of the MRs, two types of AGEs are formed: one is cross-linking and fluorescence AGEs and the other one is non-cross-linking and nonfluorescence AGEs. In addition to pentosidine and pyrraline, other types of AGEs such as CML, CEL, FFI, GOLD and MOLD, and melanoidins, as well as other compounds, which have not yet been identified, can be generated from the Amadori products. Table 1 concludes the notification, structure, and source of Maillard reaction productions in food and herb medicines.

\section{PHMRPs Analysis}

The analysis of PHMRPs is very important for quality and safety evaluation for food and herb medicines. Up to date, various methods have been developed dealing with analysis for MRPs, including pretreatment for analysis, qualitative and quantitative analysis, and structural identification analysis. First, the pretreatment for analysis includes chromatography, extraction, and membrane dialysis methods. Second, the qualitative and quantitative analysis methods include thin-layer chromatography, ultravioletvisible spectroscopy (UV spectroscopy), high-performance liquid chromatography (HPLC), gas chromatography (GC), mass spectrometry (MS), fluorescent spectrometry (FL), chemiluminescence $(\mathrm{CL})$, and electrochemical detection (ECD). In addition, structural identification and analysis methods include infrared spectrum (IR), MS, and nuclear magnetic resonance (NMR). The analysis of MRPs in food and herb medicines on the current status and the achievements made are included, the existing challenges are addressed, and the future perspectives are provided.

3.1. The Pretreatment for PHMRPs Analysis. At present, the MRPs, especially the PHMRPs in food and herb medicines, cannot be analyzed and detected accurately; one of the most important reasons is that the complex products cannot be purified effectively to obtain the pure substances. So, the pretreatment of MRPs including extraction, separation, and purification is very important for detection. Because most of the MRPs are water-soluble ingredients, the extraction methods include water extraction or water solvent extraction [15].

The separation of MRPs includes chromatography [16], extraction, dialysis, and other methods. Chromatography is a separate and determination method used by isolated MRPs with the differences of physical and chemical properties, such as adsorption, molecular shape, size, molecular affinity, and partition coefficient. Soldo et al. [17] applied a LC method combining with the taste dilution analysis and succeeded in identifying the potential bitter suppressing candidate.

Extraction usually transfers MRPs from one solvent to another by using the different solubility in different solvents. Extraction generally includes liquid-liquid extraction, solidphase microextraction, and accelerated solvent extraction. Zhang et al. [7] studied the different levels of MRPs in different extract conditions such as aqueous extract, ethanol extract, infusion, decoction, and sterilization. Combined with LH-20 separation, and absorption of UV measurement at $420 \mathrm{~nm}$ indicates that steam sterilization can lead to the formation of MRPs [18]. Shi et al. developed the method to extract acrylamide in heat-processed Chinese herb Radix Asparagi using water, and the result indicated that the extract efficiency was good [19]. Zhou and Zheng [20] employed the accelerated solvent extraction (ASE) method 

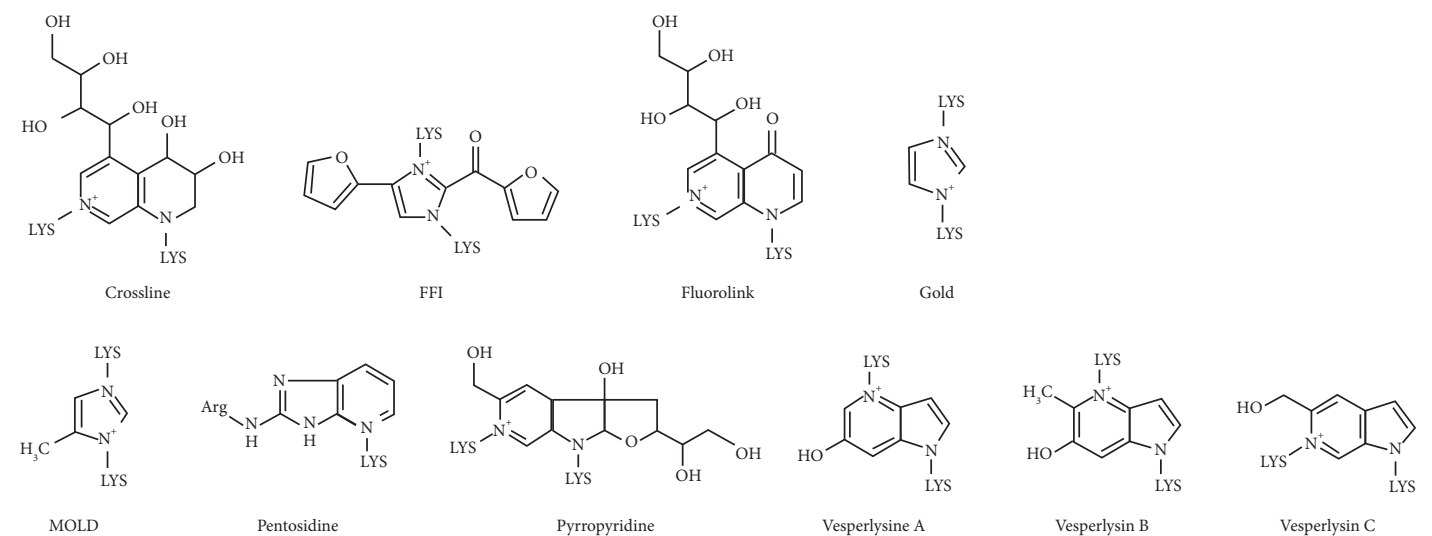

(a)
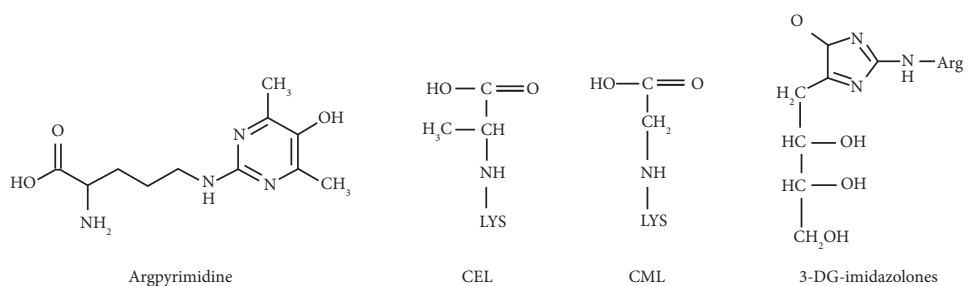

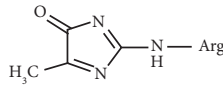

MG-imidazolones

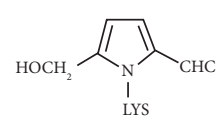

Pyrraline

(b)

Figure 1: Chemical structures of the two types of ages: (a) fluorescence and cross-linking ages and (b) nonfluorescence and non-crosslinking ages [10].

to extract 5-hydroxymethyl furaldehyde (5-HMF) in coked Angelica sinensis. Compared with traditional extraction techniques such as reflux extraction and ultrasonic extraction, ASE is more convenient, more effective, and quicker. The method can also be used for other MRPs analyses in food and herb medicines, providing a new efficient, fast, safe, and energy-saving extracting method for components analysis in food and herb medicines.

MRPs flow by a semipermeable membrane by a dialysis method, and the unreacted raw materials and the small molecules flow by the opposite side, which could remove effectively other substances from MRPs. The MRPs can be selected with different molecular weights by different dialysis modalities so that the molecular structure and the composition can be analyzed accurately. Melanoidins generated in the reactions of glucose and maltose with glycine (MW $>12500$ and 3500) was studied by microanalysis. Finally, the relationship between the molecular weight of the Melanoidins and the elemental composition of amino acids and sugars was discussed [21]. Cämmerer et al. [22] used molecular weights of 12000-14000 Da cellulose dialysis membranes to analyze the MRPs before and after the dialysis, respectively.

\subsection{The Analysis of PHMRPs}

3.2.1. The Qualitative and Quantitative Analysis Methods. Up to now, the qualitative and quantitative analysis methods for PHMRPs include thin-layer chromatography, UV-Vis spectrometry, HPLC, GC, MS, FL, CL, and electrochemical method. Table 2 lists the qualitative analysis of Maillard reaction productions in Chinese herbal medicine materials and food.

3.2.2. TLC. TLC is a qualitative analysis method which compared their retention time (Rf). Jia et al. [36] reported 5HMF was separated from the cibot rhizome for the first time. The silica gel 60 F254 TLC plate was coated by ascending development in a solution of the cyclohexane-ethyl acetateacetone with a ratio of $3: 3: 1$. The results showed that the established methods had good reproducibility and the spots were distinct.

3.2.3. UV Spectroscopy. The chromophoric groups, molecular structure, and extinction property in MRPs could be obtained using the UV-vis spectroscopy method. The UV absorbance at 280 and $420 \mathrm{~nm}$ is due to the formation of initial MRPs and the AGEs (e.g., melanoidins) for MRPs individually. Obulesu and Bhattacharya [39] investigated the color changes of the different stages for Tamarind pulp fruit development and storage, and the relationship between the UV absorption properties and pharmacodynamics activity was also discussed. Yamabe et al. [40] reported the MRs model experiment using glycine and ginsenoside Re mixture to investigate the renoprotective role of MRPs from ginsenosides, and the absorbance at $420 \mathrm{~nm}$ was measured for studying the MRPs level. At the same time, the relationship between the MRPs level and the possible application as a renoprotective agent was recommended. Jia et al. [36] identified the compound by TLC as the 5-HMF and then detected by UV-vis. The results indicated that the maximum 

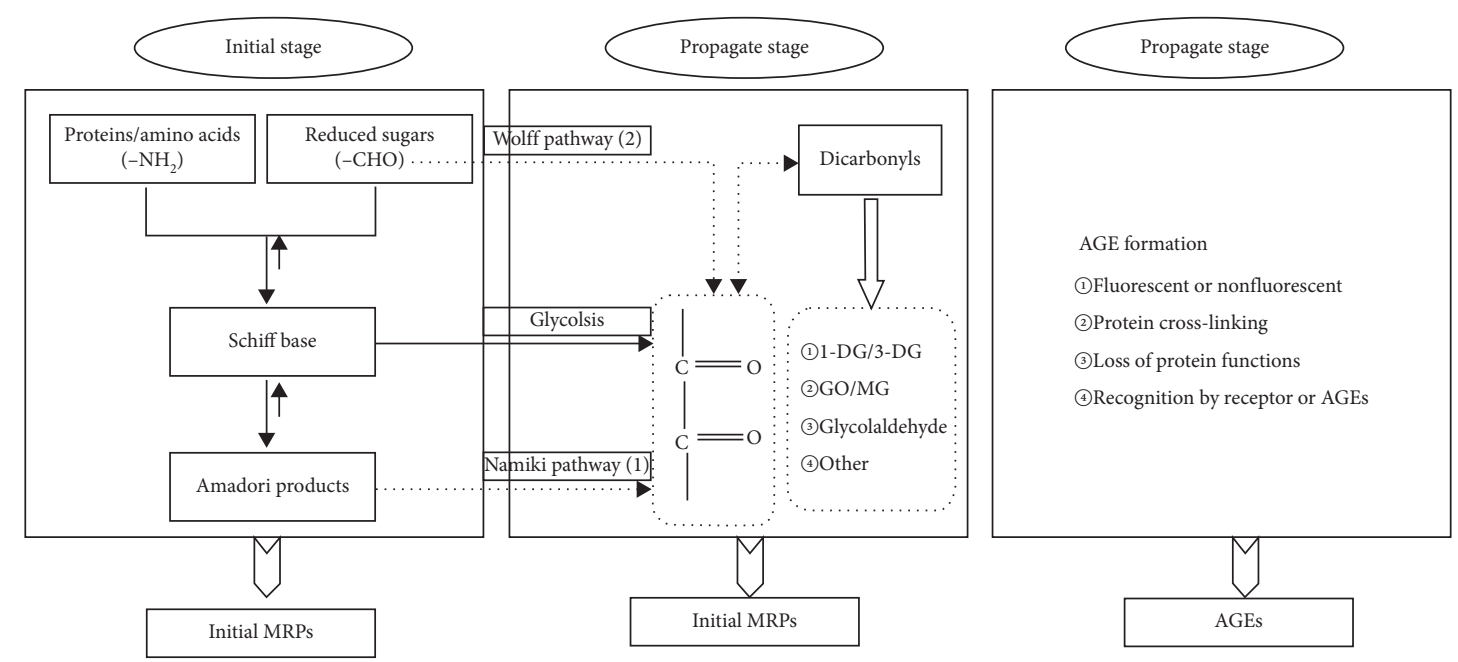

FIgURE 2: The outlines of the MPRs formation. Schiff bases are produced during reducing glucose-protein reaction. Reactive dicarbonyl precursors of ages are formed through the degradation of Schiff base intermediates (Namiki pathway) or through metal-catalyzed autoxidation of the reducing sugar (Wolff pathway) [10].

TABLE 1: The classification, nomenclature, molecular formula, structure, and source of Maillard reaction productions.

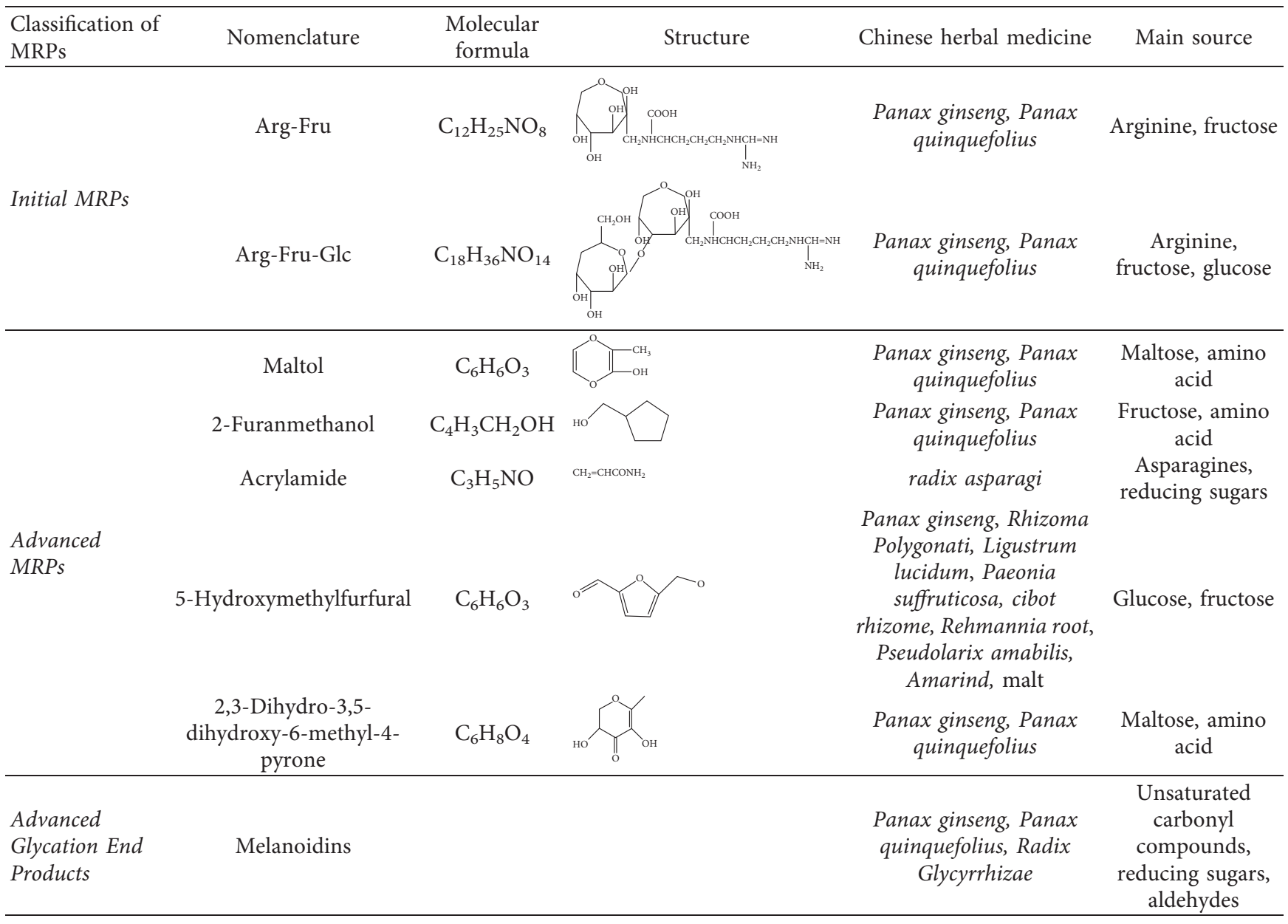

absorption wavelength was $280 \mathrm{~nm}$, indicating that the MRPs were the initial products. Yu et al. [41] first developed the capillary electrophoresis method to detect five Amadori compounds by UV at $236 \mathrm{~nm}$ without separation and derivatization based on the Amadori compound-Cu ${ }^{2+}$ complexes. The results showed that this was a convenient and reliable method for rapid analysis of the Amadori compounds. 


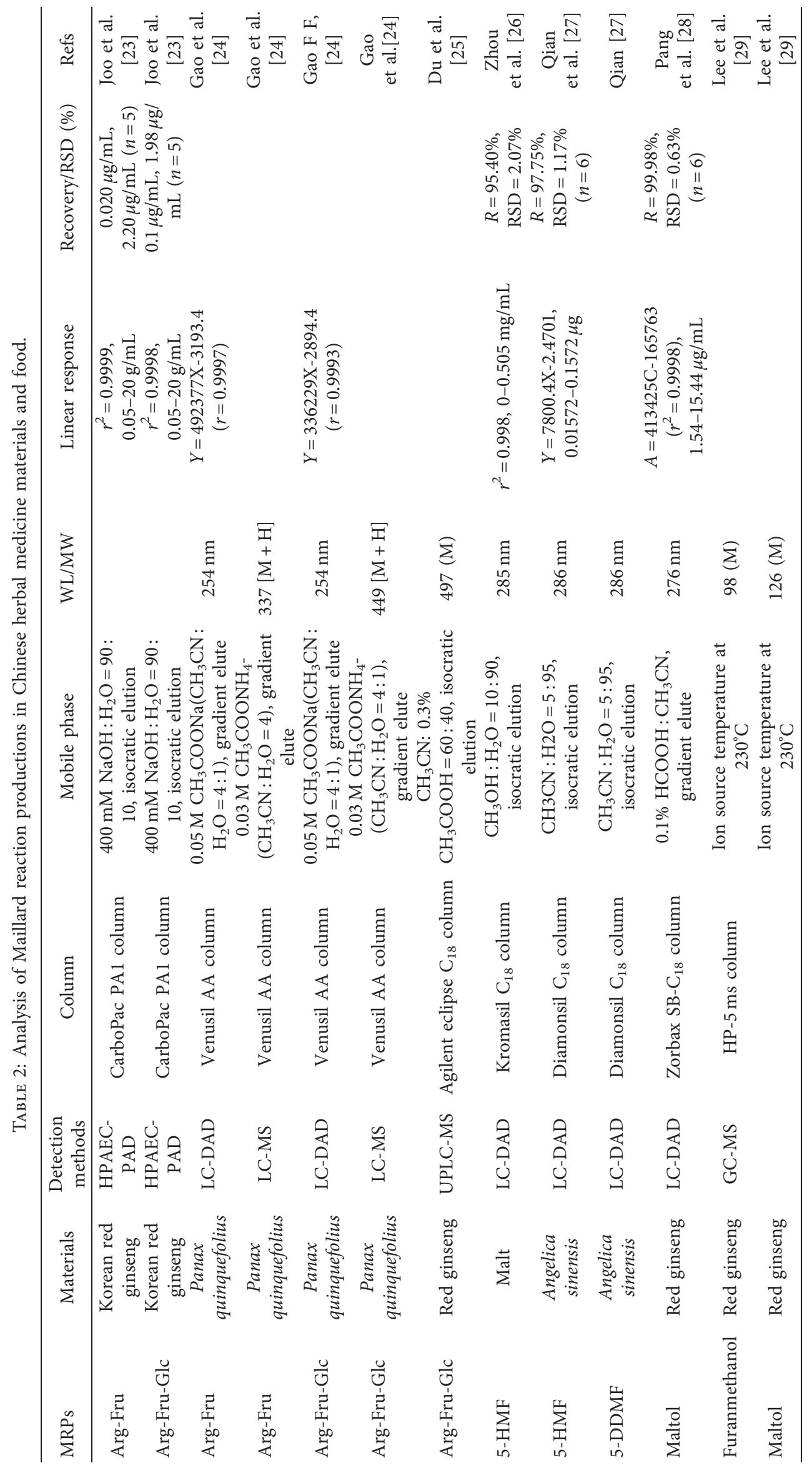




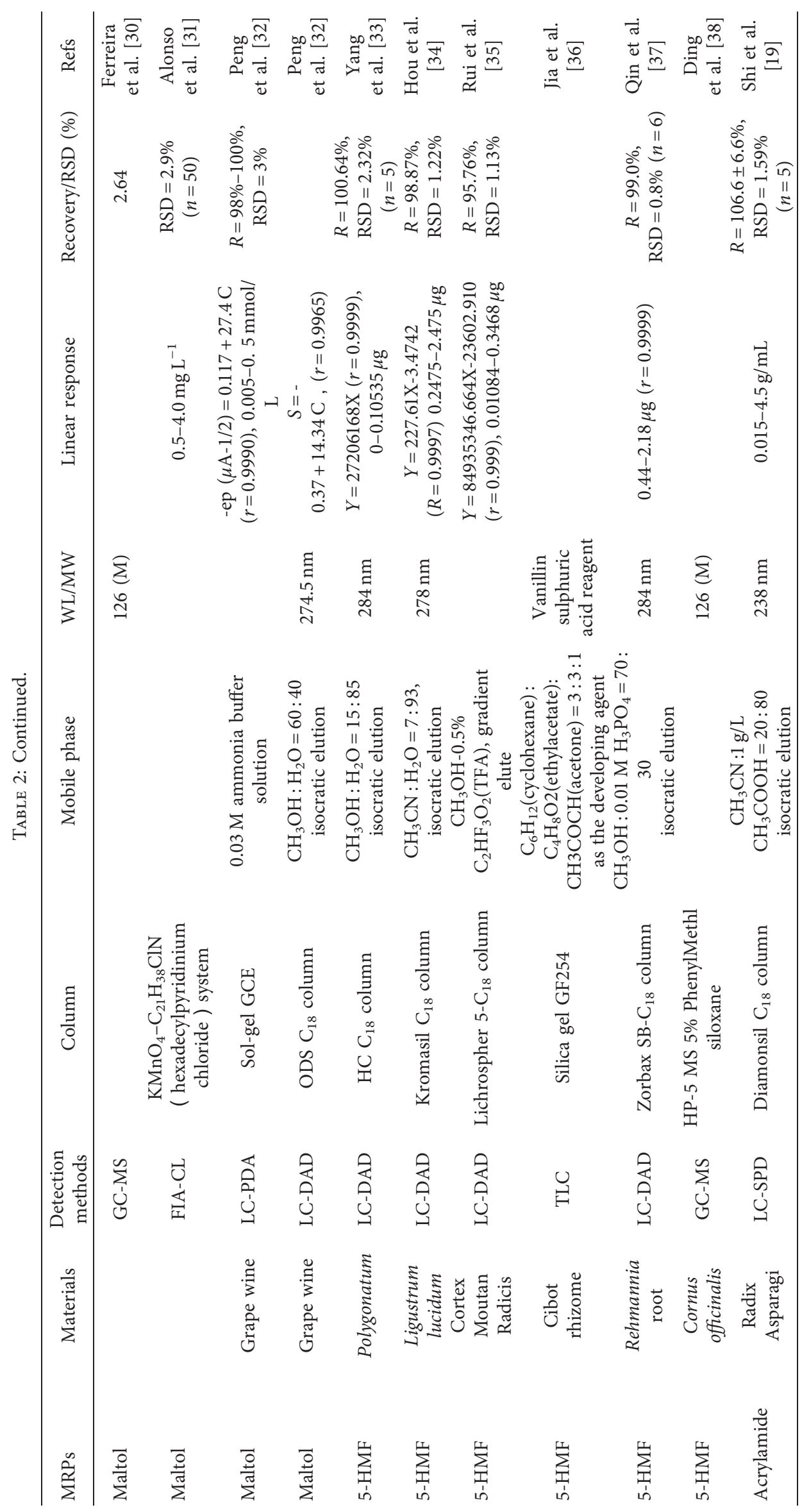


3.2.4. HPLC. HPLC is an effective method to separate and analyze components in a mixture. Gökmen [42] reported a rapid method for the simultaneous determination of HMF and patulin in apple juice. Viñas et al. [43] developed a reversed-phase gradient-elution HPLC method for the simultaneous determination of methyl anthranilate and 5HMF in honey. The proposed method can be used for both the quality evaluation of honey based on the methyl anthranilate and the quality control based on the 5-HMF.

3.2.5. GC. GC is a good tool to analyze the vaporized compounds without decomposition. Venskutonis et al. [44] used the headspace and gas sensor techniques to detect volatiles (Maillard reaction productions) in the model system. The methods can be used for assessing the volatile compounds generated during the thermal browning process of the glucose-glycine model system. Lojzova et al. [45] analyzed substituted pyrazines and other volatile aromatic compounds formed during MRs in potato chips by an alternative GC-MS method (including gas chromatography-ion trap mass spectrometry, gas chromatography-time-of-flight mass spectrometry, and comprehensive two-dimensional gas chromatography-time-of-flight mass spectrometry), both having the good effect.

3.2.6. MS. MS is a common analytical technique based on their mass-to-charge ratio. Cagliero et al. [46] described a polymeric ionic liquid sorbent coating in direct immersion solid-phase microextraction method for the analysis (especially trace-level) of AA in coffee powder. Pollien et al. [47] investigated the formation of AA during the thermal treatment $\left(120-170^{\circ} \mathrm{C}\right)$ of potato as well as in Maillard model systems (composing reducing sugars and asparagine). It was achieved by online monitoring of AA released into the headspace of the samples using proton transfer reaction mass spectrometry. It was found that the level of AA released into the headspace during thermal treatment of potato was positively correlated to temperature.

3.2.7. Chemiluminescence. Chemiluminescence is the emission of light produced in certain chemical reactions without light, heat, or electromagnetic excitation. Lakeev et al. [48] investigated the complex fluctuation dynamics of the MR, that is, a multistage reaction between a carbonylcontaining compound and the nucleophilic amino group of an amino-containing compound at different temperatures, as visualized by chemiluminescence.

3.2.8. Electrochemical Method. The electrochemical method can be employed as a useful tool for the analysis of Maillard intermediates and final products. Galvanic potentials serve as useful indicators for reductones, Amadori compounds, and pyranones analyzed readily by HPLC-ECD techniques. Electrochemical methods provide valuable noninvasive probes to follow the stages of the Maillard reaction and for investigating its mechanism [49]. Rizzi et al. [50] investigated the electrochemical properties of $\beta$-alanine/ carbohydrate MRPs using a combination platinum/Ag- $\mathrm{AgCl}$ $\left(\mathrm{Cl}^{-}\right)$redox electrode. Joo et al. [23] detected and quantified redox-active Maillard reaction intermediates and products by a variety of electrochemical techniques. Amperometry in the form of electrochemical detectors was used in conjunction with chromatographic separation to analyze Amadori compounds and pyranones.

3.2.9. Fluorescent Spectrum. Trevisan et al. [51] explored the fluorescent method to investigate the effect of cooking conditions on MRPs in beef. It was found that fluorescence intensity changed consistent with the results of FML levels.

$\mathrm{Hu}$ et al. [52] reported a fluorescent sensing method based on AA polymerization and the unique photo-physical properties of quantum dots (QDs) detecting AA [53, 54]. The correlation was established between the concentrations of AA and changes of fluorescence intensities after UV irradiation can be used as the base of the detection of AA. The lower sensitivity of this method limits it to be used for detecting AA in various food samples.

3.3. Structure Identification Analysis of PHMRPs. At present, the methods of structure identification analysis for MRPs in food and herb medicines are mainly including infrared spectrum (IR spectrum), nuclear magnetic resonance (NMR), and MS.

3.3.1. IR Spectrum. Because the functional groups are generally associated with the IR spectrum frequencies, it can be used to determine the structures of the compounds or to determine the functional groups. IR spectroscopy is one of the main means to study the structures of organic molecules and has been applied to analyze the molecular structures of the MRPs. Gullón et al. [55] used the IR spectrum to analyze the functional groups and evaluate the introduction of glucose into the chitosan molecule. After reaction, it can be observed that the absorption bands of Chit showed changes from $1697 \mathrm{~cm}^{-1}$ to $1596 \mathrm{~cm}^{-1}$ (C-N double bond), which suggesting it was formed Schiff base between the reducing termination of Glc and the amino groups of Chit [56].

3.3.2. NMR. The most commonly studied nuclei are ${ }^{1} \mathrm{H}$ and

${ }^{13} \mathrm{C}$ NMR. ${ }^{1} \mathrm{H}$ NMR was used to monitor ligand-exchange reactions on the compounds, and ${ }^{13} \mathrm{C}$ NMR was used to investigate the structures of the compounds. Gullón et al. [55] used ${ }^{1} \mathrm{H}$ NMR to analyze the functional groups and study the reaction between glucose and chitosan. Comparing the spectra of the original glucose and chitosan, one of the most important differences is the new signals appearing at $2.09 \mathrm{ppm}$, indicating the new formation of $\mathrm{NH}-\mathrm{CH}_{2}$ (Amadori product). Wu [57] studied the effect of gamma irradiation on the browning of depolymerized chitosan; the result showed that gamma irradiation was an effective technology to inhibit browning during the depolymerization of chitosan. Lima-Dellamora et al. [18] found that the sodium bisulfite added could cause the absorbance of 
Echinodorus grandiflorus at $420 \mathrm{~nm}$ dropped comparison with the control.

3.3.3. MS. MS is an analytical tool that makes use of the mass-to-charge ratio $(\mathrm{m} / \mathrm{z})$ of particles to determine the molecular formula of a compound. In the lab, the tool is very powerful in making sure of the known and unknown compounds [58]. MS is a key tool to explore the reactions and the different reaction products in food and herb medical field. [58]. Pollien et al. [47] established a PTR-MS method to monitor AA formation online during Maillard reaction systems and processed food. The PTR-MS ion signal at $\mathrm{m} / \mathrm{z}$ 72 was shown to be exclusively due to protonated AA obtained without fragmentation. Kislinger et al. [59] used matrix-assisted laser desorption/ionization time of flight mass spectrometry (MALDI-TOF MS) to qualitatively study the formation of early Maillard products of lysozyme, produced upon incubation with seven different sugars (Dribose, L-rhamnose, D-glucose, D-fructose, D-galactose, D-lactose, and D-maltose) in solution, in the presence of oxygen. The report first reported the use of MALDI-TOF MS peptide mapping as a quick and highly selective method for the detection of early-stage Maillard products produced upon incubation of lysozyme with seven different reducing sugars. Linda et al. [60] prepared MRPs by the reaction of 5hydroxymethylfurfural (5-HMF) separately with glucose and glycine. The spectra display two responses (one for each isotope) that are separated by a mass number that directly corresponds to the number of nitrogen atoms present in the MRP.

\section{PHMRPs Control}

The Maillard reaction paths taking place depend strongly on $\mathrm{pH}$, temperature, and the properties of the reactants (e.g., types of sugar, amino acid, and protein) [61]. It is acknowledged that, in the case of proteins or peptides, the reactive amino groups are available for the $M R$ or the Strecker reaction. In the case of proteins, the MR often leads to cross-link formation [62]. Deoxyosones are considered essential intermediates for the general MRs [63]. An alternative view on the MRs has been given by Yaylayan [64] who considers the initial stage as three primary fragmentation pools arising from sugars, amino acids, and Amadori/Heyns products. Lysine is the major amino acid that contributes to MRs as it has a free amino group that can readily react with reducing sugars [65]. Recent evidence suggests that AGEs have a propensity to generate reactive oxygen species (ROS) [66]. Furthermore, glucose and other aldehydes, free or protein-bound, can undergo autooxidation reactions and generate radicals and other reactive intermediates (e.g., $\mathrm{H}_{2} \mathrm{O}_{2}$ and other peroxides) that contribute to AGE formation. Ma et al. [67] reported that acylation of antioxidant of bamboo leaves with fatty acids by lipase and the acylated derivatives' efficiency in the inhibition of acrylamide formation in fried potato crisps. Wu [57] investigated the effect of oxygen and $\mathrm{pH}$ on the browning of chitosan exposed to gamma radiation. It was found that oxygen and $\mathrm{pH}$ value could play important roles in inhibiting browning of irradiated chitosan. Przygodzka et al. [68] reported that the furosine was decreased in cakes with cloves, allspice, spice mix, and vanilla, which will provide a base for the application.

\section{Conclusions and Future Research Outlook}

During the past few years, it has been of concern that partial MRPs in food and medicines are harmful to the human body because they have been proven to be closely related to a variety of diseases such as chronic diabetes, Parkinson's disease, Alzheimer's disease, and aging and also induce cancer and heritable mutations in laboratory animals. Many contributions reported the formation, analysis, and control of PHMRPs in food and herb medicines; with also some progress being achieved.

As for the outlook of future research, an acknowledged fact is that PHMPRs in the MRs need to be more adequately demonstrated. Overtly, the formation, analysis, and control of PHMPRs in food and Chinese herbal medicine started late, and the structures of the MRPs are complex and varied. First, the preliminary research on the formation of PHMPRs only stays in a single factor or a macrolevel. However, if not studying their formation from a molecular level, it cannot accurately reveal the PHMPRs formation regulation from the raw material, processing conditions, and chemical microenvironment. Second, the separation, purification, qualitative and quantitative analysis, and structure analysis are of great difficulty because of the complexity of the PHMPRs. Furthermore, the contents of some MRPs are very low and cannot be easy to separate, and various related factors bring many challenges to the analysis of PHMPRs in food and Chinese herbal medicines. In addition, the analysis of MRPs is far behind the analysis of sugar, amino acid, and protein in food and Chinese herbal medicines. There have been many theoretical and practical problems to be solved and most of the analysis is still in the laboratory stage of exploration and really can't be used in actual application, far from being able to meet the urgent need for people's understanding of its structure and function.

In conclusion, first, we study the PHMPRs formation from a molecular level. Second, we establish a high sensitivity and high accuracy analysis method that is an important issue in the analysis of PHMPRs in the future, with the development of various analytical techniques in the separation and analysis of PHMPRs in pharmacology and other fields. Third, the preliminary research on the formation of PHMPRs limited effective directional control of PHMPRs in food and medicines. MR is consecutive and parallel reaction steps, which is very complex. Therefore, fundamental studies, especially kinetic research during the progress of the $M R$, need to be further investigated. On the other hand, the optimized processing parameters are important factors to minimize AGEs formation. The ultimate challenge will be to achieve a substantial reduction of PHMPRs while keeping desirable product attributes such as flavor and color, which are generated by similar MR pathways. 


\section{Conflicts of Interest}

The authors declare that they have no conflicts of interest.

\section{Authors' Contributions}

Yali Li designed the manuscript and contributed to the writing of the manuscript. Yanzhu Zhu, Peihe zheng, Zhengyi Qu, Hao Zhang, Xiangmin Piao, and Yingping wang contributed to the proofreading of the manuscript. Wei Hou supported the paper to be published. All the authors read and proofed the final manuscript.

\section{Acknowledgments}

The authors sincerely thank the project of the Jilin Provincial Department of Science and Technology China (no. 20190201160JC), the Jilin Province Development and Reform Commission (2019C052-10), the National Science Foundation for Youths of China (no. 31401606), the Innovation Project of Medicinal Plant Germplasm Resources and Breeding Innovation Team, the Chinese Academy of Agricultural Sciences (no. CAAS-ASTIP-2016-ISAPS), the Project of International Cooperation and Exchanges (no. 2015DFA31290), and the Central Public-Interest Scientific Institution Basal Research Fund (no. 1610342020008).

\section{References}

[1] S. I. F. S. Martins, W. M. F. Jongen, and M. A. J. S. Van Boekel, "A review of maillard reaction in food and implications to kinetic modelling," Trends in Food Science and Technology, vol. 11, pp. 364-373, 2001.

[2] J. E Hodge, "Chemistry of browning reactions in model systems," Journal of Agricultural and Food Chemistry, vol. 1, pp. 928-943, 1953.

[3] Y. L. Li, X. M. Piao, S. F. Pang, Z. Y. Qu, and Y. P. Wang, "The pre-treatment and chromatography analysis of amino acids in chinese herbal medicine," Special Wild Economic Animal and Plant Research, vol. 3, pp. 112-117, 2019.

[4] Y. F. Wang, C. A. Jin, H. Zhao, J. Y. Liu, and F. Wang, "Determination of saponins in ginseng, red ginseng and American ginseng by Uplc-Esi-Ms/ms," Special Wild Economic Animal and Plant Research, vol. 3, pp. 58-66, 2019.

[5] C. Harris, L.-P. Beaulieu, M.-H. Fraser et al., "Inhibition of advanced glycation end product formation by medicinal plant extracts correlates with phenolic metabolites and antioxidant activity," Planta Medica, vol. 77, no. 02, pp. 196-204, 2011.

[6] A. W. Stitt, "Advanced glycation: an important pathological event in diabetic and age related ocular disease," British Journal of Ophthalmology, vol. 85, no. 6, pp. 746-753, 2001.

[7] J. Zhang, Z. Mei, X. Huang, Y. Ding, Y. Liang, and Y. Mei, "Inhibition of Maillard reaction in production of low-molecular-weight chitosan by enzymatic hydrolysis," Carbohydrate Polymers, vol. 236, pp. 116059-116066, 2020.

[8] G. M??nch, C. E. Shepherd, H. Mccann et al., "Intraneuronal advanced glycation endproducts in presenilin-1 Alzheimer??s disease," Neuroreport, vol. 13, no. 5, pp. 601-604, 2002.

[9] J. W. Baynes, "The maillard hypothesis on aging: time to focus on DNA," Annals of the New York Academy of Sciences, vol. 959, no. 1, pp. 360-367, 2002.
[10] C.-H. Wu, S.-M. Huang, J.-A. Lin, and G.-C. Yen, "Inhibition of advanced glycation endproduct formation by foodstuffs," Food \& Function, vol. 2, no. 5, pp. 224-234, 2011.

[11] M. A. R. Mazumder, P. Hongsprabhas, and R. T. Vasudevan, "In vitro and in vivo inhibition of maillard reaction products using amino acids, modified proteins, vitamins, and genistein: a review," Journal of Food Biochemistry, vol. 43, no. 1, pp. 13089-13095, 2019.

[12] F. C. De Oliveira, J. S. D. R. Coimbra, E. B. D. Oliveira et al., "Food protein-polysaccharide conjugates obtained via the maillard reaction: a review," Critical Reviews in Food Science and Nutrition, vol. 56, no. 7, pp. 1108-1125, 2016.

[13] K. Mikulikova, I. Miksik, and Z. Deyl, "Non-enzymatic posttranslational modifications of bovine serum albumin by oxo-compounds investigated by chromatographic and electrophoretic methods," Journal of Chromatography B, vol. 815, no. 1-2, pp. 315-331, 2005.

[14] A. Ardestani and R. Yazdanparast, "Inhibitory effects of ethyl acetate extract of Teucrium polium on in vitro protein glycoxidation," Food and Chemical Toxicology, vol. 45, no. 12, pp. 2402-2411, 2007.

[15] Z. Dai, I. Nemet, W. Shen, and V. M. Monnier, "isolation, purification and characterization of histidino-threosidine, a novel maillard reaction protein crosslink from threose, lysine and histidine," Archives of Biochemistry and Biophysics, vol. 463, no. 1, pp. 78-88, 2007.

[16] C. Helou, P. Jacolot, C. Niquet-Léridon, P. Gadonna-Widehem, and F. J. Tessier, "Maillard reaction products in bread: a novel semi-quantitative method for evaluating melanoidins in bread," Food Chemistry, vol. 190, pp. 904-911, 2016.

[17] T. Soldo and T. Hofmann, "Application of hydrophilic interaction liquid chromatography/comparative taste dilution analysis for identification of a bitter inhibitor by a combinatorial approach based on maillard reaction chemistry," Journal of Agricultural and Food Chemistry, vol. 53, no. 23, pp. 9165-9171, 2005.

[18] E. C. Lima-Dellamora, K. C. V. Waldhelm, A. M. Alves, C. A. S. Lage, A. A. C. Leitão, and R. M. Kuster, "Genotoxic maillard byproducts in current phytopharmaceutical preparations of echinodorus grandiflorus," Anais da Academia Brasileira de Ciências, vol. 86, no. 3, pp. 1385-1394, 2014.

[19] Z. Shi, H. Zhang, and X. Zhao, "Ultrasonic-assisted precolumn derivatization-HPLC determination of acrylamide formed in Radix Asparagi during heating process," Journal of Pharmaceutical and Biomedical Analysis, vol. 49, no. 4, pp. 1045-1047, 2009.

[20] G. F. Zhou and S. J. Zheng, "Optimization of accelerated solvent extraction for 5-hmf in coke angelicae sinensis with response surface analysis," Chinese Archives of Traditional Chinese Medicine, vol. 29, no. 8, pp. 1900-1903, 2011.

[21] S. Mundt and B. L. Wedzicha, "Comparative study of the composition of melanoidins from glucose and maltose," Journal of Agricultural and Food Chemistry, vol. 52, no. 13, pp. 4256-4260, 2004.

[22] B. Cämmerer, L. W. Kroh, R. Boukherroub, and O. Melnyk, "Investigation of the influence of reaction conditions on the elementary composition of melanoidins," Food Chemistry, vol. 53, no. 1, pp. 55-59, 1995.

[23] K. M. Joo, C. W. Park, H. J. Jeong, S. J. Lee, and I. S. Chang, "Simultaneous determination of two amadori compounds in Korean red ginseng (panax ginseng) extracts and rat plasma by high-performance anion-exchange chromatography with pulsed amperometric detection," Journal of Chromatography $B$, vol. 865, no. 1-2, pp. 159-166, 2008. 
[24] F.-F. Gao, W.-Y. Zhang, L.-M. Liu et al., "Detection and distribution of arginine derivatives in Panax quinquefolius L. and investigations of their antioxidant properties," LWT, vol. 49, no. 1, pp. 34-41, 2012.

[25] Q.-Q. Du, S.-Y. Liu, R.-F. Xu, M. Li, F.-R. Song, and Z.-Q. Liu, "Studies on structures and activities of initial maillard reaction products by electrospray ionisation mass spectrometry combined with liquid chromatography in processing of red ginseng," Food Chemistry, vol. 135, no. 2, pp. 832-838, 2012.

[26] Z. Y. Zhou, X. Chen, K. Xu, D. Y. Shi, C. X. Zhao, and G. J. Jiang, "Determination of 5-hydroxymethyl furfural by Hplc," Food Industry, vol. 36, no. 8, pp. 270-273, 2015.

[27] X. D Qian and G. F Zhou, "Study on content change rules of polysaccharide and two chemical constituents after processing in angelica sinensis from different sources," Chinese Archives Traditional Chinese Medicine, vol. 32, no. 6, pp. 1320-1323, 2014.

[28] X. Q. Pang, Z. L. Ye, D. K. Li, and D. Z. Zhou, "Study on the methods for detection of hydrolyzate of crotonoside-isoguanine in stewed soup," Shaanxi Journal of Traditional Chinese Medicine, vol. 34, no. 11, pp. 1542-1543, 2013.

[29] S.-J. Lee, T. W. Moon, and J. Lee, "Increases of 2-furanmethanol and maltol in Korean red ginseng during explosive puffing process," Journal of Food Science, vol. 75, no. 2, pp. c147-C151, 2010.

[30] V. Ferreira, I. Jarauta, R. López, and J. Cacho, "quantitative determination of sotolon, maltol and free furaneol in wine by solid-phase extraction and gas chromatography-ion-trap mass spectrometry," Journal of Chromatography A, vol. 1010, pp. 95-103, 2003.

[31] S. Alonso, M. C. Lahuerta, L. Zamora, and C. Martinez, "determination of the flavor enhancer maltol through a fiadirect chemiluminescence procedure," Journal of Analytica Chimical Acta, vol. 438, no. 1-2, pp. 157-163, 2001.

[32] S. H. Peng, W. H. Ma, and J. W. Di, "Determination of maltol and ethyl maltol by spectrophotometry, hplc and voltammetry," Chinese Journal of Spectroscopy Laboratory, vol. 22, no. 4, pp. 680-682, 2005.

[33] Y. Yang, M. Xu, Y. X. Feng, and H. H. He, "Analysis of 5hydroxymethylfurfural in sublimate of cibot rhizome," Chinese Journal of Chinese Material Medicine, vol. 31, no. 1, pp. 17-19, 2008.

[34] J. Hou and X. L. Zhang, "Effects of processing on the contents of polysaccharide and 5-hydroxymethylfurfural in ligustrum lucidum ait," Chinese Traditional Patent Medicine, vol. 31, no. 4, pp. 572-575, 2009.

[35] H. B. Rui, X. L. Zhao, X. Z. Tan, and W. Li, "Content variation of 5-hydroxymethylfurfural in charred paeonia suffruticosa of various degree of processing," Hebei Journal of Traditional Chinese Medicine, vol. 32, no. 9, pp. 1399-1400, 2010.

[36] T. Z. Jia, H. L. Chen, S. Q. Xie, H. Zhou, and G. Y. Li, "analysis of 5-hydroxymethylfurfural in sublimate of cibot rhizome," Chinese Traditional Patent Medicine, vol. 24, no. 10, pp. 768-771, 2002.

[37] X. Y. Qin, J. Zhou, X. Y. Li, and X. L. Sun, "RP-HPLC determination of 5-hydroxymethylfurfural in prepared rehmannia root," Fourth Military Medical University, vol. 27, no. 6, pp. 510-511, 2006.

[38] X. Ding and M. Y. Wang, "Studies on separation, appraisal and the biological activity of 5-hmf in comus officinalis," Chinese Journal of Chinese Material Medica, vol. 33, pp. 392-396, 2008.

[39] M. Obulesu and S. Bhattacharya, "Color changes of tamarind (tamarindus indical.) pulp during fruit development, ripening, and storage," International Journal of Food Properties, vol. 14, no. 3, pp. 538-549, 2011.

[40] N. Yamabe, K.-I. Song, W.-J. Lee et al., "Chemical and free radical-scavenging activity changes of ginsenoside Re by maillard reaction and its possible use as a renoprotective agent," Journal of Ginseng Research, vol. 36, no. 3, pp. 256-262, 2012.

[41] J. Yu, W. Aboshora, S. Zhang, and L. Zhang, "Direct UV determination of Amadori compounds using ligand-exchange and sweeping capillary electrophoresis," Analytical and Bioanalytical Chemistry, vol. 408, no. 6, pp. 1657-1666, 2016.

[42] V. Gökmen and J. Acar, "Simultaneous determination of 5hydroxymethylfurfural and patulin in apple juice by reversedphase liquid chromatography," Journal of Chromatography A, vol. 847, no. 1-2, pp. 69-74, 1999.

[43] P. Viñas, N. Campillo, and M. H. Córdoba, "Simultaneous liquid chromatographic analysis of 5-(hydroxymethyl)-2furaldehyde and methyl anthranilate in honey," Food Chemical, vol. 44, no. 44, pp. 67-72, 1992.

[44] R. P. Venskutonis, R. Vasiliauskait, A. Galdikas, and A. Šetkus, "Use of GC-headspace and "electronic nose" for the detection of volatile compounds from glucose-glycine Maillard reaction," Food Control, vol. 13, no. 1, pp. 13-21, 2002.

[45] L. Lojzova, K. Riddellova, J. Hajslova, J. Zrostlikova, J. Schurek, and T. Cajka, "Alternative gc-ms approaches in the analysis of substituted pyrazines and other volatile aromatic compounds formed during maillard reaction in potato chips," Analytica Chimica Acta, vol. 641, no. 1-2, pp. 101-109, 2009.

[46] C. Cagliero, T. D. Ho, C. Zhang, C. Bicchi, and J. L. Anderson, "Determination of acrylamide in brewed coffee and coffee powder using polymeric ionic liquid-based sorbent coatings in solid-phase microextraction coupled to gas chromatography-mass spectrometry," Journal of Chromatography A, vol. 1449, pp. 2-7, 2016.

[47] P. Pollien, C. Lindinger, C. Yeretzian, and I. Blank, "Proton transfer reaction mass spectrometry, a tool for on-line monitoring of acrylamide formation in the headspace of maillard reaction systems and processed food," Analytical Chemistry, vol. 75, no. 20, pp. 5488-5494, 2003.

[48] S. G. Lakeev, V. L. Voeikov, and S. F. Timashev, "Fluctuation dynamics of chemiluminescence in the maillard reaction," Russian Journal of General Chemistry, vol. 81, no. 1, pp. 234-242, 2011.

[49] G. P. Rizzi, "Electrochemical aspects of the maillard reaction and related reactions," Food Reviews International, vol. 29, no. 2, pp. 178-191, 2013.

[50] G. P. Rizzi, "Electrochemical study of the maillard reaction," Journal of Agricultural and Food Chemistry, vol. 51, no. 6, pp. 1728-1731, 2003.

[51] A. J. B. Trevisan, D. De Almeida Lima, G. R. Sampaio, R. A. M. Soares, and D. H. Markowicz Bastos, "Influence of home cooking conditions on maillard reaction products in beef," Food Chemistry, vol. 196, pp. 161-169, 2016.

[52] Q. Hu, X. Xu, Z. Li et al., "Detection of acrylamide in potato chips using a fluorescent sensing method based on acrylamide polymerization-induced distance increase between quantum dots," Biosensors and Bioelectronics, vol. 54, pp. 64-71, 2014.

[53] J. Liu, X. Yang, K. Wang, R. Yang, and C. Wu, "A switchable fluorescent quantum dot probe based on aggregation/disaggregation mechanism," Chemical Communications, vol. 47, no. 3, pp. 935-937, 2010.

[54] C. Tansakul, E. Lilie, E. D. Walter et al., "Distance-dependent fluorescence quenching and binding of CdSe quantum dots by functionalized nitroxide radicals," The Journal of Physical Chemistry C, vol. 114, no. 17, pp. 7793-7805, 2010. 
[55] B. Gullón, M. I. Montenegro, A. I. Ruiz-Matute, A. CardelleCobas, N. Corzo, and M. E. Pintado, "Synthesis, optimization and structural characterization of a chitosan-glucose derivative obtained by the Maillard reaction," Carbohydrate Polymers, vol. 137, pp. 382-389, 2016.

[56] X. Liu, Z. Feng, N. B. Harris, J. D. Cirillo, H. Bercovier, and R. G. Barletta, "Identification of a secreted superoxide dismutase inMycobacterium aviumssp.paratuberculosis," FEMS Microbiology Letters, vol. 202, no. 2, pp. 233-238, 2001.

[57] Y. Wu, "Prevention of browning of depolymerized chitosan obtained by gamma irradiation," carbohydrate polymers, vol. 101, pp. 857-863, 2014.

[58] L. Fay and H. Brevard, "Contribution of mass spectrometry to the study of the maillard reaction in food," Mass Spectrometry Reviews, vol. 24, no. 4, pp. 487-507, 2005.

[59] T. Kislinger, A. Humeny, S. Seeber, C.-M. Becker, and M. Pischetsrieder, "Qualitative determination of early maillard-products by maldi-tof mass spectrometry peptide mapping," European Food Research and Technology, vol. 215, no. 1 , pp. 65-71, 2002.

[60] M. B. Linda, S. Naylor, and J. T. Andy, "Investigation of maillard reaction products using $15 \mathrm{n}$ isotope studies and analysis by electrospray ionization-mass spectrometry," Food Chemistry, vol. 62, no. 2, pp. 179-183, 1998.

[61] W. Jian, J. He, Y. Sun, and J. Pang, "Comparative studies on physicochemical properties of bovine serum albumin-glucose and bovine serum albumin-mannose conjugates formed via Maillard reaction," LWT-Food Science and Technology, vol. 69, pp. 358-364, 2016.

[62] C. M. J. Brands, B. L. Wedzicha, and M. A. J. S. Van Boekel, "Quantification of Melanoidin Concentration in Sugar-Casein Systems," Journal of Agricultural and Food Chemistry, vol. 50, no. 5, pp. 1178-1183, 2002.

[63] L. F. Marvin, V. Parisod, L. B. Fay, and P. A. Guy, "Characterization of lactosylated proteins of infant formula powders using two-dimensional gel electrophoresis and nanoelectrospray mass spectrometry," Electrophoresis, vol. 23, no. 15, pp. 2505-2512, 2002.

[64] V. A. Yaylayan, "Classification of the Maillard reaction: a conceptual approach," Trends in Food Science \& Technology, vol. 8, no. 1, pp. 13-18, 1997.

[65] H. Li, H. Yang, P. Li et al., "Maillard reaction products with furan ring, like furosine, cause kidney injury through triggering ferroptosis pathway," Food Chemistry, vol. 319, pp. 12368-12377, 2020.

[66] D. Bonnefont-Rousselot, "Glucose and reactive oxygen species," Current Opinion in Clinical Nutrition and Metabolic Care, vol. 5, no. 5, pp. 561-568, 2002.

[67] X. Ma, E. P. Wang, Y. Y. Lu, Y. Wang, S. Y. Ou, and R. Yan, "Acylation of antioxidant of bamboo leaves with fatty acids by lipase and the acylated derivatives' efficiency in the inhibition of acrylamide formation in fried potato crisps," PLOS ONE, vol. 10, no. 6, pp. 1-11, 2015.

[68] M. Przygodzka, H. Zieliński, Z. Ciesarová, K. Kukurová, and G. Lamparski, "Study on sensory quality, antioxidant properties, and maillard reaction products formation in ryebuckwheat cakes enhanced with selected spices," Journal of Chemistry, vol. 2015, pp. 1-9, 2015. 